misleading statement. There actually were three lots of cows. Then do you imply that the returns which are sent by the Local Authorities cannot be correct?-I have no doubt that they are correct to this extent, that that is the date on which Professor M'Call put them down, but my explanation of the case is a very different one. There were actually three lots of cows in that man's premises; that is to say, there was the lot that I inoculated; there was a lot that was left, simply because they were unfit for inoculation and were subsequently inoculated; and there was the lot that he purchased in to be inoculated immediately after the outbreak was reported.

I want to ask you whether any of the cattle inoculated on February 9th showed the disease on April 9th?-Yes, they did; but they were reported by me, not very many days after the inoculation, to the owner. I have over and over again said that it must not be held that, because cattle do not exhibit the disease at the time of inoculation and examination, they are free from it ; but I say this, that before a given time they will exhibit it. These animals did exhibit it, and they were pointed out by me to the owner for him to do his part; that is to say, bring them to Mr M'Call's notice. He never did so, and I do not think Mr M'Call was attending the byres as closely as I was; I was in the byres every other day almost.

Mr BOWEN-JONES.-Do you know of any cases where inoculation has taken successfully more than once on the same animal?-No, I say it is impossible. - Is inoculation always effectual?-Yes; inoculation will undoubtedly always arrest and stamp out pleuro-pneumonia.

In the next number of this Journal I shall refer in the first place to the objections which Mr Rutherford has taken to my evidence, and in the second place I shall give in detail the history of a recent outbreak of pleuro-pneumonia on the same premises, and I shall by post-mortem and other evidence prove that Mr Rutherford's inoculation of Salmond's stock in February 1887 not only did not arrest the spread of the disease at that time, but that it actually carried it on, and was the cause of a second outbreak in June of the present year.

\title{
FARCY OF THE OX ${ }^{1}$
}

\section{By M. E. NocARD, Director of the Alfort Veterinary School.}

THE older authors, Hutrel d'Arboval, ${ }^{2}$ Gellé, ${ }^{3}$ Cruzel, ${ }^{4}$ have described under the name of "farcy of the ox" (farcin du bauf) a chronic malady characterised by suppurative inflammation of the superficial lymphatic vessels and glands, rarely fatal, but ultimately rendering itself evident by emaciation, and "by symptoms of tubercular phthisis" (Cruzel).

This affection, formerly frequent, if one may judge by the rather numerous observations contained in the first volumes of the Recueil de Medecine Veterinaire, appears to have become very rare to-day, at least in France. Indeed, Zundell, in his re-edition of Hutrel d'Arboval, $^{5}$ and Peuch, in that of Cruzel's work, ${ }^{6}$ do not mention it. 
However, according to what several confrères have told me, it must exist still in Bresse and in the north of France, and it certainly exists at Guadeloupe, where, according to $M$. Couzin, ${ }^{1}$ it is very frequent, and despite its invariably slow evolution (it lasts ordinarily several years) much more grave than in France, death occurring after a longer or shorter period. Farcy of the ox, it would be superfluous to insist upon the point, has nothing in common with the glanderous farcy of the Equidæ; the experiments long since carried out by Renault, and those more recent by Cadeac and Malet, have demonstrated in a peremptory manner that the ox is absolutely refractory to inoculation with the virus of glanders.

But although it is certain that farcy of the ox is something other than a manifestation of glanders, there has not hitherto been any certainty regarding its real nature. In the article "Diseases of the Lymphatics" in M.H. Bouley's Dictionary, I have emitted the hypothesis -based on the description of it given by authors, for I have never myself had the opportunity to observe it - that it was probably of the nature of a tuberculous lymphangitis; that was also the opinion of M. Couzin, who has seen many affected animals, and made numerous autopsies.

That was an error. M. Couzin having sent me some pus collected pure, and some fragments of the diseased tissues, I have been able to assure myself that farcy of the ox is a microbian malady very different from tuberculosis.

I. Symptoms, progress, and lesions of the disease.-The following description, which I borrow from Cruzel, will give a good idea of the symptoms and progress of the affection:-

"Farcy of the ox has its seat ordinarily on the limbs and under the belly along the track of the subcutaneous vessels. It presents itself under the form of circumscribed tumours and cords; and in the same animal when the farcy exists on two limbs one remarks occasionally the two different forms. The cords or tumours are indolent, sometimes very hard, at other times slightly fluctuating; they are found on both faces of the cannon, on the fore-arm and on the inner surface of the thigh; they are always directed towards the lymphatic glands, which are usually engorged.

"When the cords or buttons of farcy exist on the cannon or on the fore-arm, it is rarely that the lymphatic glands placed in front of the shoulder are not more or less engorged, and it is equally rare that symptoms of tubercular phthisis do not manifest themselves when there is some fary of the extrenities and when this ganglionic engorgement is apparent. One encounters on the course of the cords some circumscribed abscesses which are perfectly delineated, although in many cases the cords are moist and fluctuating.

"The farcy abscesses sometimes burst, especially at the bend of the knee and the hock. In incising the cords or tumours one can squeeze out by compressing the tumour or the cord at its base a whitish material having some resemblance to thick cream. This matter is inodorous.

"The farcy develops itself insensibly in the ox, and its duration, consequently, is very prolonged. I have seen oxen that had carried farcy tumours for several years without manifesting any other morbid 
symptom. They had worked, been fattened, and sent to the abattoir just as if they had not been the subject of farcy.

"The farcy tumours do not terminate by resolution in the ox, and suppuration is a permanent state, modified only by induration."

The foregoing symptomatic picture agrees fully with the observations collected in France. However, from the point of view of prognosis, it appears a little too optimistic; for, according to Maillet, "the affected animals are fattened with difficulty, and the owners therefore get quit of them sooner or later when they perceive that emaciation persists in spite of the care taken to put them in good condition." Moreover, if it is true, as Cruzel says, that "ultimately the affected animals present symptoms of tubercular phthisis," it is difficult to admit with him that they can "work, fatten, and reach the abattoir quite as well as if they were not attacked with farcy."

M. Couzin has observed in several oxen at Guadeloupe more acute symptoms. The affection seems to begin by inflammation of a lymphatic gland; the brachial, prescapular, and prepectoral glands are the earliest and the most frequently attacked; the glands become tumefied, hot, painful, œdematous ; they augment slowly in volume, and ultimately present the character of a cold indurated abscess; puncture gives exit to a thick, creamy, occasionally caseous and grumous pus. The wall of the abscess is extremely thick $(8,10$, or I 2 centimetres), indurated, lardaceous, creaking under the stroke of the bistoury, and its inner face is downy, soft to the touch, and pimply. After puncture, the subject seems to return to health; but after a longer or shorter period, sometimes six months, other tumours appear and run through the same phases as the first, and the animal dies in a state of marasmus.

It is interesting to note that none of the French veterinarians who have described farcy of the ox appear to have had occasion to make the autopsy of an affected animal. M. Couzin has been more fortunate; in all the autopsies that he has made, besides the purulent collections which were developed in the glands of the trunk and members, he has found the lungs, the liver, the spleen, and the glands crammed with pseudo-tubercles, the central part of which had undergone the caseous or purulent transformation, according to the volume of the tumour (unpublished communication).

2. Micro-biological study of the disease.-As I have already said, M. Couzin procured for me some pus and pieces of organs obtained at the autopsy of an ox that had been farcied for two years. The pus had been aspirated pure in slender tubes, sterilised and closed at the lamp; the pieces were preserved as small fragments in strong alcohol.

Examination of the pus, practised after Ehrlich's process by double staining with methyl-blue or Bismark brown, eliminates at the outset the hypothesis of a tubercular lesion. Koch's bacillus is absent, and there is not observed any other microbe that has fixed the secondary stain. Gram's method gives the same negative result when one decolorises thoroughly in the alcohol; but if one decolorises with aniline oil, according to the indications of Weigert, a very different result is obtained. Among the pus cells, coloured red by eosine or carmine, there appears in considerable numbers a special microbe, 
different from any hitherto described. It is a slender long bacillus presenting itself under the form of small masses interwoven in an inextricable fashion, the central part resembling an opaque nucleus from which a myriad of fine prolongations radiate to the periphery, the majority of them appearing ramified. One might compare the whole to a head of cauliflower, a faggot of thorns, or a burr.

In respect of dimensions, this bacillus may be compared to that of rouget du porc.

The examination of the diseased tissues (lungs, liver, spleen, glands) shows them crammed with tubercle-like nodules, the central part of which, purulent rather than caseous, presents in great quantity the same bacillary masses in the form of brushwood.

The culture of this microbe is effected easily in all the liquid or solid media maintained in contact with the air, at a temperature varying between $30^{\circ}$ and $40^{\circ} \mathrm{C}$. Tubes of gelatine peptone do not give any trace of growth at the temperature of the room; if they are placed in the incubator the seed multiplies in a few days.

The pus collected pure from the centre of an abscess or from a pseudo-tubercle serves admirably to start a culture; it does not contain any other microbe than the bacillus described above.

On gelose, the microbe develops in small irregularly rounded masses, salient, opaque, thicker at the edges, of a yellowish white tint, mammillated, dull and powdery-like; at length these patches, of a lichenoid aspect, unite and become confounded, giving to the whole culture the appearance of a thick coarsely folded membrane.

On potato, the culture grows rapidly under the form of small scaly patches, very salient and dry, pale yellow in colour, the edges, as if cut perpendicularly, seeming to be raised above the level of the substratum.

On gelatinised serum, the culture is less rapid; but it has the same aspect as on the gelose, save that it is more humid. In the different bouillons, it is again under the form of irregular masses that the bacillus multiplies. The majority of these whitish masses fall to the bottom of the flask, but some remain floating at the surface, where they spread as a sort of pellicle, rounded and lenticular in form, dirty grey in colour, with a greenish reflection, powdery in appearance, and not allowing itself to be wet by the liquid. It is especially in bouillon with glycerine and peptone added that the culture takes this aspect; one might compare it then to leaves of the water-lily spreading themselves at the surface of a pond, or better still, to soup in which the fat drops have been solidified by cooling.

The culture succeeds still, less abundantly and less rapidly, in the media whose reaction is slightly acid. It does not appear to modify the reaction of neutral or alkaline bouillon, even when sugar has been added to them.

Sown in milk the microbe develops with the same characters, without provoking its coagulation or modifying the reaction.

The organism is exclusively ærobic; all attempts to grow it in a vacuum or in carbonic acid have failed.

Whatever be the medium of culture, the microscopic examination shows that the microbe has reproduced itself there in affecting the same disposition as in the living tissues; there are always the same filamentous masses, interwoven in an inextricable fashion, of which the 
bacillary nature is not appreciable except at the margins, where the irradiations have again the ramifying aspect that I have mentioned above.

This ramification is more apparent than real ; the attentive study of recent colonies shows that the development of the filaments is effected by elongation; when the bacillus has acquired the double of its primitive length it divides, and the newly-formed segment becomes inflected, generally at a right angle to the original element, which continues to grow in a straight line; in short, it is a case of false dichotomy analogous to that observed in the Cladothrix; but the study of it is rendered difficult by the small dimensions of the microbe, and its inaptitude to fix most of the aniline colours. Moreover, the coloration by Gram's method is coarse, and not suited for the definition of morphological particulars of microbes.

The older colonies appear rich in spores, especially those that have developed at the surface of the glycerine liquids; the spores, extremely small, resist impregnation by colouring matters; they appear under the form of ovoid uncoloured lacuna at the ends of the bacillary segments, at the level of the points where the dichotomisation appears to take place.

The cultures preserve their virulence and their vitality for a long time ; after a sojourn of four months in an incubator at $40^{\circ}$, they grow with the same vigour in the different media, and the guinea-pigs inoculated from them die as rapidly as at the outset.

Heating does not appear to me to kill the bacillus unless it is pushed above $65^{\circ}$. At $65^{\circ}$ an old culture heated for I 5 minutes has given new cultures, and killed the guinea-pigs inoculated by intraperitoneal injection; Io minutes heating at $70^{\circ}$ has destroyed the microbe's virulence and power of vegetation.

3. Pathogenic action of the microbe.-The bacillus of farcy of the ox is inoculable; but the results of the inoculation vary greatly according to the species of animal to which one has had recourse, and especially according to the method of inoculation practised.

In general terms, it is to the guinea-pig that it is necessary to resort by preference; then come the ox and the sheep. The rabbit, the dog, the cat, the horse, and the ass may be considered refractory.

Intraperitoneal and intravenous injection provoke constantly in the guinea-pig, after a delay of 9 to $20^{\circ}$ days, lesions that may be mistaken for those of miliary tuberculosis.

On opening a guinea-pig inoculated by the peritoneum, the serous membrane shows itself literally crammed with tubercle-like nodules, at the centre of which the microbe has multiplied, presenting itself always under the aspect of bacillary masses in the form of brushwood; these nodules are especially confluent in the omentum, which is transformed into a sort of voluminous mammillated pudding; pressure forces out some droplets of puriform matter, which is thick and difficult to dissociate, and which shows under the microscope a considerable quantity of bacillary masses.

The viscera of the abdominal cavity (liver, spleen, kidneys, intestines) appear equally studded with pseudo-tubercles; but an attentive examination shows that only their peritoneal envelope is attacked, their parenchyma is quite intact. The organs of the thoracic cavity are never invaded. 
The lesions consecutive to intravenous injection simulate to a still greater degree the lesions of a general miliary tuberculosis; at the autopsy of the subject of experiment all the viscera, but especially the lung, the liver, and the spleen, are found infiltrated with a considerable number of small tubercle-like nodules, at the centre of which a microscopic examination, after staining by the Gram-Weigert method, shows one or several tufts of bacilli.

Whether pus or liquid of culture has been injected is unimportant ; the result is always the same: the lesions are identical ; death supervenes always equally soon. In the cow and in the sheep intravenous injection provokes analogous lesions, equally generalised throughout the soft organs; but the animals resist so long that I cannot yet say if death might be the consequence.

Two old cows and two Breton sheep were inoculated on the 26 th March $\mathrm{I} 888$ by the injection into the jugular of a dilution of pus taken from a guinea-pig.

One of the cows had to be killed on the I Ith April to serve for the exercises of operative medicine; at the autopsy, the lungs showed a considerable number of grey, semi-transparent, miliary granulations, especially abundant in the superficial layers. Each of these had for its centre a large tuft of interwoven bacilli. The liver and the spleen presented similar lesions, but in small numbers.

One of the sheep killed on the 22nd April had identical lesions, but much more acute.

The two other subjects are still living; they have appeared well throughout.

In the rabbit, the dog, the cat, the horse, and the ass, the intravascular or intraperitoneal injections provoke no accident, at whatever date one kills the subjects of experiment after the inoculation; whatever be the dose of the product injected, pus or culture, no trace of the microbe is found. The hypodermic inoculation realises almost the conditions of natural infection, in the sense that even in the guinea-pig the lesion provoked progresses with extreme slowness.

At the point of inoculation, in all the subjects, an abscess of which the pus is very rich in microbes forms, but while this abscess remains comparatively small it bursts, empties itself, and cicatrises rapidly in the animals specifically refractory (rabbit, dog, horse, ass); it persists in the others ; in the sheep and cow it remains small, ulcerates from time to time, becomes indurated, and then seems to disappear; but several weeks or months afterwards a new abscess shows itself in the neighbourhood, at a point nearer the centre than the first. Without doubt it would require many months, and perhaps several years, for the microbe, always present in the pus, to gain the viscera and become generalised.

In the guinea-pig the lesions provoked by hypodermic inoculation are more grave and rapid. The abscess which forms at the point of inoculation is always voluminous; in a few days the lymphatic vessels and glands of the region become engorged, indurated, and the seat of an enormous phlegmon which ulcerates and discharges several cubic centimetres of pus; at this moment it seems as if the animal, very much emaciated, must soon succumb; but, on the contrary, it returns gradually to the normal state, it improves in condition, and retains of the grave lesion which it presented only an induration of the lymphatic vessels and glands primitively attacked. 
Of 16 guinea-pigs inoculated under the skin, I died on the 54 th day with a miliary pseudo-tuberculosis generalised to all the viscera; all the others have resisted; their general state is excellent; and those that I have killed $\mathrm{I}, 2,3$, and 4 months after the inoculation had no lesion except those of the glands and vessels that collect the lymph from the region of the inoculation: small purulent foci rich in bacillary masses which have conserved their virulence, surrounded by a considerable induration.

On that account I am inclined to believe that in the guinea-pig in which subcutaneous inoculation provoked generalised lesions a vein must have been perforated and have received a small quantity of the inoculated liquid.

To sum up, experimentation confirms to the full the data of clinical experience. The bacillus of farcy of the ox injected into the veins or into the peritoneum is fatal to the guinea-pig in a few days; on the contrary, when inoculated under the skin of the same animal it remains for a long time confined to the neighbouring lymphatic glands or vessels, without modifying the general state of the subject, without interfering with its fattening.

From that one comprehends that farcied cattle may be able to continue their services as long as the bacillus has not passed the lymphatic glands, and germinated in the viscera.

\section{ANTHRAX IN SWINE.}

By Professor Edgar Crookshank, M.B., of King's College, London.

Researches communicated to the Pathological Section at the Annual Meeting of the British Medical Association held in Glasgow, August 1888 .

HISTORICAL.-There has been considerable diversity of opinion as to the occurrence of anthrax in swine. Some of the early writers on the diseases of animals speak of anthrax being communicated to pigs; but whether the outbreaks were in all cases examples of true anthrax, or indeed whether the disease referred to was in any single instance anthrax, has long been a subject of controversy. It is well known that many diseases have been confounded under the name anthrax, and many authorities even up to the time of the present inquiry have maintained that the disease which we now recognise as being produced by the anthrax bacillus could not be communicated to swine. In support of these statements I may be allowed to quote from various writers on the subject.

Fleming, in the "Manual of Veterinary Sanitary. Science and Police," writing on Anthrax and Anthracoid Diseases, informs us that Shrœekius described a serious outbreak in Augsburg in I 7 I 2 in which horses, cattle, pigs, geese, fowls and such wild animals as deer and boars died in immense numbers, and that Gilbert maintained that dogs, hogs, birds, and wild creatures died in large numbers from ingesting the flesh of animals which had perished from anthrax. It is even stated that Gilbert had seen a pig and eight young hogs die 